\title{
El consumo de drogas como una práctica cultural dentro de las pandillas
}

\author{
Francisco Rafael Guzmán-Facundo ${ }^{1}$ \\ Luiz Jorge Pedrão ${ }^{2}$ \\ Karla Selene Lopez-García ${ }^{3}$ \\ María Magdalena Alonso-Castillo ${ }^{3}$ \\ Santiaga Enriqueta Esparza-Almanza ${ }^{4}$
}

\begin{abstract}
Actualmente el fenómeno social del narcotráfico y la violencia relacionada a la drogas ha minimizado las implicaciones del consumo de drogas en pandillas. Este artículo se basa en entrevistas semi-estructuradas a profundidad de un estudio realizado con jóvenes de pandillas del área Metropolitana de Nuevo León, México, con el objetivo de reflexionar y analizar el consumo de drogas como práctica cultural dentro de las pandillas. En la búsqueda del significado, lo primero que se muestra es el inicio de los miembros de pandillas en el consumo de drogas, y se describe la forma en que las drogas le son presentadas por la familia y/o los amigos miembros de la pandilla. Posteriormente se describe el significado del uso de drogas en la vida cotidiana de las pandillas y se observa en qué medida su uso es aceptable y normalizado.
\end{abstract}

Descriptores: Drogas Ilícitas; Factores de Riesgo; Consumidores de Drogas; Adolescente; Jóvenes.

\footnotetext{
${ }^{1}$ Profesor Doctor, Facultad de Enfermería, Universidad Autónoma de Nuevo León, Nuevo León, México. E-mail: pako2001@hotmail.com.

2 Enfermero, Doctor en Enfermería. Profesor Doctor, Escola de Enfermagem de Ribeirão Preto, Universidade de São Paulo, Centro Colaborador de la OMS para el Desarrollo de la Investigación en Enfermería, SP, Brasil. E-mail: lujope@eerp.usp.br.

3 Profesora Doctora, Facultad de Enfermería, Universidad Autónoma de Nuevo León, Nuevo León, México. E-mail: Karla: kslg2001@hotmail.com, María Magdalena: magdalena_alonso@hotmail.com.

${ }^{4}$ Profesora MCE, Facultad de Enfermería, Universidad Autónoma de Nuevo León, Nuevo León, México. E-mail: sesparza54@yahoo.com.mx.
}

Correspondencia:

Luiz Jorge Pedrão

Universidade de São Paulo. Escola de Enfermagem de Ribeirão Preto

Departamento de Enfermagem Psiquiátrica e Ciências Humanas

Av. dos Bandeirantes, 3900

Bairro: Monte Alegre

CEP: 14040-902 Ribeirão Preto, SP, Brasil

E-mail: lujope@eerp.usp.br 


\section{O uso de drogas como prática cultural dentro de gangues}

O fenômeno social do narcotráfico e da violência, relacionada às drogas, tende, atualmente, a minimizar os envolvimentos do consumo de drogas em gangues. Esse artigo se baseia em entrevistas, aprofundadas, de estudo realizado com jovens de gangues, na área Metropolitana de Nuevo León, México, com o objetivo de refletir e analisar o consumo de drogas como prática cultural, dentro das gangues. Na busca do significado, a primeira coisa mostrada é o início dos membros de gangues no uso de drogas, e se descreve a forma em que as drogas são apresentadas pela família e pelos amigos, membros das gangues. Em seguida, descreve-se o significado do uso de drogas na vida cotidiana e mostra até que ponto sua utilização é aceitável e normal.

Descritores: Drogas Ilícitas; Fatores de Risco; Consumidores de drogas; Adolescente.

\section{Drugs Use as a Cultural Practice Within Gangs}

Today, the social phenomenon of drugs trafficking and violence related to drugs has tended to minimize the implications of drugs consumption in gangs. This article is based on in-depth interviews in young gangs in the metropolitan area of Nuevo León, Mexico, with a view to reflecting on and analyzing the drug as a cultural practice within gangs. In the search for meaning, the first thing that is shown is the beginning of gang members in drugs consumption, and the form how drugs are presented by family members and friends of the gang is described. Next, we described the meaning of drugs use in everyday life and show the extent to which drugs use is acceptable and normalized.

Descriptors: Street Drugs; Risk Factors; Drug Users; Adolescent.

\section{Introducción}

Para algunos jóvenes y adolescentes, experimentar el consumo de drogas ilícitas es un rito necesario para atravesar la fase de individuación. De hecho, se podría decir que la mayor parte de estos jóvenes son experimentadores o consumidores ocasionales. Ellos, influenciados generalmente por pares, hacen uso de sustancias de manera ocasional y no necesariamente desarrollan dependencia. Sin embargo, para aquellos jóvenes que pertenecen a pandillas, el consumo de drogas constituye a menudo la única alternativa para enfrentarse al mundo en el que viven, así mismo, pueden ser los más sensibles a reaccionar favorablemente a la dependencia de drogas $^{(1-2)}$.

Durante la última década se muestra un enorme crecimiento en la literatura científica y de reportajes en los medios de comunicación sobre jóvenes que se integran a pandillas. En parte, esto es el resultado del resurgimiento de pandillas juveniles en todo el país, especialmente en el norte, incluyendo Nuevo León, México. Se ha añadido a este grupo de jóvenes estereotipos de violencia, de uso y tráfico de drogas como principales actividades. Por lo tanto las pandillas de hoy son percibidas como espacios en donde las drogas y la conducta violenta van de la mano(3-4).

Actualmente el fenómeno social del narcotráfico y la preocupación de la sociedad con las pandillas específicamente con la violencia relacionada a la drogas ha tendido a minimizar la importancia e implicaciones del consumo de drogas entre los miembros de las pandillas. Hasta la fecha, la investigación sobre pandillas está centrada en el problema social que causan relacionado con actos delictivos y poco se ha realizado en el contexto social y de salud que ocasiona el consumo de drogas ilícitas en la vida de los integrantes de pandillas ${ }^{(5-7)}$.

En el norte del país específicamente en Nuevo León figuran publicaciones que han tratado de explicar la conducta del consumo de drogas en los jóvenes enfocándose a factores individuales y personales, así mismo se ha explicado el inicio del consumo de drogas a través de las etapas de adquisición, mostrando 
factores personales y familiares que influyen en el mantenimiento de la conducta ${ }^{(8-9)}$. Esta falta de interés en la investigación sobre el consumo de drogas es aún más sorprendente, dado que existe un alto número de miembros de pandillas que usan drogas ilícitas; un estudio en Nuevo León ha encontrado que cerca de $60 \%$ de los miembros de pandillas han consumido drogas ilícitas, destacando el consumo de marihuana, con la excepción del alcohol, como la sustancia más utilizada en la vida de pandillas(10).

El relativo desinterés de un análisis del contexto social del consumo de drogas se deriva no sólo de la preocupación por las pandillas y la violencia del narcotráfico, sino también es el resultado de centrarse en el uso de drogas desde una perspectiva de delincuencia. Este enfoque ha significado que los estudios de consumo de drogas dentro de las pandillas, como el campo de la investigación de drogas en general, se han concentrado principalmente en examinar la epidemiología de uso de drogas. Si bien este paradigma teórico ha sido importante para documentar la extensión del uso de drogas dentro de las pandillas, el enfoque ha ignorado, en gran medida, la cultura y el significado del consumo de drogas.

Sin pretender subestimar la importante contribución de esta literatura en nuestro entendimiento de la vida de pandillas, el objetivo de este documento es reflexionar y analizar sobre el consumo de drogas como práctica cultural dentro de las pandillas y no sólo como un problema de conducta, centrándose en aspectos de actitud y percepción de normas sobre el consumo de drogas en los estilos de vida de los miembros de pandillas.

\section{Métodos}

\section{Población, lugar y muestra}

Los participantes del estudio fueron 28 adolescentes y jóvenes marginales, hombres (23) y mujeres (5) de 16 a 24 años de pandillas juveniles del área metropolitana de la ciudad de Monterrey. El número de participantes no fue predeterminado, resultó del muestreo teórico, el cual forma parte de la metodología utilizada. La Figura 1 muestra las características generales de los participantes del estudio.

\begin{tabular}{|c|c|c|c|c|c|c|}
\hline Participante & Edad & Sexo & Ocupación & Trabajo remunerado & Estado civil & Consumo de droga actual \\
\hline $\mathrm{P} 1$ & 22 & Masculino & Albañil & $\mathrm{Si}$ & Casado & Marihuana \\
\hline P2 & 24 & Femenina & Ninguna & No & Divorciada & Marihuana \\
\hline P3 & 15 & Masculino & Ninguna & No & Soltero & $\begin{array}{l}\text { Marihuana } \\
\text { Tolueno }\end{array}$ \\
\hline P4 & 19 & Femenina & Ninguna & No & Soltera & Marihuana \\
\hline P5 & 18 & Masculino & Ninguna & No & Soltero & Tolueno \\
\hline P6 & 16 & Masculino & Ninguna & No & Soltero & Marihuana \\
\hline P7 & 15 & Masculino & Estudiante & No & Soltero & Marihuana \\
\hline P8 & 19 & Masculino & Albañil & $\mathrm{Si}$ & Divorciado & Marihuana \\
\hline P9 & 19 & Masculino & Obrero & $\mathrm{Si}$ & Soltero & Tolueno \\
\hline P10 & 17 & Masculino & Albañil & $\mathrm{Si}$ & Soltero & $\begin{array}{l}\text { Marihuana } \\
\text { Cocaína }\end{array}$ \\
\hline P11 & 17 & Masculino & Albañil & $\mathrm{Si}$ & Soltero & Marihuana \\
\hline $\mathrm{P} 12$ & 17 & Masculino & Obrero & $\mathrm{Si}$ & Soltero & Marihuana \\
\hline $\mathrm{P} 13$ & 18 & Masculino & Albañil & $\mathrm{Si}$ & Soltero & Cocaína \\
\hline P14 & 22 & Masculino & Comerciante & $\mathrm{Si}$ & Unión libre & Tolueno \\
\hline P15 & 16 & Masculino & Estudiante & No & Soltero & $\begin{array}{l}\text { Marihuana } \\
\text { Tolueno }\end{array}$ \\
\hline P16 & 19 & Masculino & Obrero & $\mathrm{Si}$ & Soltero & $\begin{array}{c}\text { Marihuana } \\
\text { Resistol }\end{array}$ \\
\hline P17 & 17 & Femenina & Ninguna & No & Soltera & Tolueno \\
\hline P18 & 18 & Femenina & Empleada & $\mathrm{Si}$ & Soltera & Marihuana \\
\hline P19 & 28 & Masculino & Chofer & $\mathrm{Si}$ & Unión Libre & $\begin{array}{l}\text { Cocaína } \\
\text { Tolueno }\end{array}$ \\
\hline $\mathrm{P} 20$ & 16 & Femenina & Estudia & No & Soltera & Cocaína \\
\hline P21 & 20 & Masculino & Limpieza & $\mathrm{Si}$ & Soltero & Tolueno \\
\hline P22 & 20 & Masculino & Lavacoches & $\mathrm{Si}$ & Soltero & Marihuana \\
\hline P23 & 27 & Masculino & Ninguna & No & Soltero & Tolueno \\
\hline P24 & 19 & Masculino & Obrero & $\mathrm{Si}$ & Casado & $\begin{array}{c}\text { Cocaína } \\
\text { Marihuana }\end{array}$ \\
\hline P25 & 18 & Masculino & Ninguna & No & Soltero & $\begin{array}{c}\text { Cocaína } \\
\text { Marihuana }\end{array}$ \\
\hline
\end{tabular}




\begin{tabular}{|c|c|c|c|c|c|c|}
\hline Participante & Edad & Sexo & Ocupación & Trabajo remunerado & Estado civil & Consumo de droga actual \\
\hline P26 & 16 & Masculino & Estudia y trabaja & $\mathrm{Si}$ & Soltero & $\begin{array}{c}\text { Marihuana } \\
\text { cocaína }\end{array}$ \\
\hline P27 & 16 & Masculino & Ninguna & No & Soltero & $\begin{array}{c}\text { Tolueno } \\
\text { Marihuana }\end{array}$ \\
\hline P28 & 17 & Masculino & Empleado & $\mathrm{Si}$ & Soltero & $\begin{array}{c}\text { Marihuana } \\
\text { Cocaína }\end{array}$ \\
\hline
\end{tabular}

Figura 1 - Características generales de los participantes del estudio

\section{Instrumento de recolección de datos}

Para cumplir el objetivo, se utilizó una entrevista a profundidad, se utilizó un equipo de audio grabador digital y notas de campo. La entrevista se inició con la siguiente pregunta: ¿Cómo es para ti usar o consumir drogas?; esta dio dirección a los puntos del estudio que fueron explorados y nuevas preguntas se realizaron con el fin de esclarecer y fundamentar las experiencias.

\section{Procedimiento de recolección de datos}

Para la recolección de los datos, los sujetos fueron visitados en las horas en que se reúnen, generalmente por las noches después de las 20 horas en su lugar de reunión. Para el acercamiento con los sujetos del estudio, se realizaron visitas a barrios urbanos marginados, donde se observan grupo de pandillas juveniles, la detección de grupos se realizó por referencias de informantes claves tales como, vecinos de los barrios marginados.

Para el trabajo de campo se conformó un equipo de trabajo por cuatro profesionales de enfermería que recibieron capacitación y entrenamiento en el manejo de la metodología, así como en la selección de la muestra, uno de los investigadores se desempeñó como supervisor, encargado de organizar y observar el trabajo de campo a fin de intervenir en imprevistos que pudieran interferir en las entrevistas. El tiempo para cada entrevista no fue predeterminado, varió de 20 a 60 minutos, la mayoría de las entrevistas se realizó en condiciones ideales de privacidad. Aquellas entrevistas en donde hubo menor privacidad ocurrieron cuando otros compañeros del entrevistado se acercaban para conocer lo que se estaba realizando, sin embargo, el miembro del equipo que desempeñaba la función de supervisor se acercaba sutilmente para retirarlos y dar la información que ellos solicitaban.

Todas las entrevistas fueron grabadas con el consentimiento previo de los participantes, los cuales no mostraron inhibición por la presencia de la grabadora. Las entrevistas se realizaban los días lunes, miércoles y viernes por las noches, con el fin de tener tiempo, el día siguiente, para la trascripción de las mismas, lo que permitió recordar comportamientos verbales y no verbales ocurridos e iniciar algunas reflexiones que maximizaron la oportunidad de descubrir variaciones entre los conceptos.

\section{Análisis de los datos}

El análisis se basó en la metodología de la Teoría Fundamentada en los Datos ${ }^{(11)}$, la cual hunde sus raíces en el Interaccionismo Simbólico, en donde el investigador intenta determinar qué significado simbólico tienen los objetos para los grupos sociales y cómo interactúan unos con otros. Desde esta perspectiva se trató de construir como los pandilleros ven el consumo de drogas en su realidad social. El enfoque del que parte la Teoría Fundamentada es descubrir, conceptos, hipótesis y proposiciones partiendo directamente de los datos, a través de una comparación constante (es decir se codifican y analizan las narraciones simultáneamente para desarrollar conceptos) y de un muestreo teórico (hasta agotar las variaciones de los conceptos identificados).

El primer paso que permitió el desarrollo de la Teoría Fundamentada en los Datos es la trascripción de las entrevistas, seguido del procedimiento de codificación. El procedimiento de codificación se realizó de acuerdo con la literatura específica(11), donde se presenta en tres etapas: codificación abierta, codificación axial y codificación selectiva.

La codificación abierta es el proceso analítico por medio del cual se identificaron los conceptos (categorías) $y$ se descubrieron en los datos sus propiedades $y$ dimensiones. Para identificar, descubrir, y desarrollar los conceptos fue necesario abrir el texto y exponer los pensamientos e ideas contenidos en él. Para realizar esta codificación se utilizó el programa de computación ATLAS/ti 5, el cual es una herramienta que permitió organizar los datos, durante la codificación abierta, los datos se descomponen en partes discretas, se 
examinan minuciosamente y se comparan en busca de similitudes y diferencias. Los acontecimientos, sucesos, objetos y acciones o interacciones que se consideraron conceptualmente similares relacionados con el significado del consumo de drogas se agrupan bajo conceptos más abstractos, denominándolos categorías.

La codificación axial es el proceso donde se relacionaron las categorías con las subcategorías; es denominado axial porque la codificación ocurre alrededor del eje de una categoría, y enlaza las categorías en cuanto a sus propiedades y dimensiones. El propósito de la codificación axial fue comenzar el proceso de reagrupar los datos que se fracturaron durante la codificación abierta. En la codificación axial, las categorías se relacionan con sus subcategorías para formar explicaciones más precisas y completas sobre el significado del consumo de drogas en los jóvenes de pandillas (fenómeno de interés).

Finalmente la codificación selectiva es el proceso que permite integrar y refinar la explicación del fenómeno de interés. El primer paso para la integración es determinar la categoría central, la cual representa el tema principal de la investigación, en este caso se identificaron los significados del consumo de drogas donde se resaltan actitudes positivas y normas que favorecen el consumo.

\section{Consideraciones éticas}

El presente estudio se realizó de acuerdo a lo dispuesto en el Reglamento de la Ley General de Salud en Materia de Investigación para la Salud en México(12). Se consideró, lo establecido en asegurar el respeto a la dignidad de los sujetos y la protección de sus derechos y bienestar, señalando que podían retirarse del estudio en cualquier momento sin ningún tipo de problema para ellos. Se obtuvo el consentimiento informado de los sujetos de la investigación y el estudio fue aprobado por el Comité de Ética de la Facultad de Enfermería de la Universidad de Nuevo León, México.

\section{Resultados}

El inicio del consumo de drogas en jóvenes de pandillas está dado por condiciones causales, en las que sobresalen: la influencia familiar, la influencia de integrantes de la banda y el vivir la experiencia inicial.

\section{Influencia familiar}

El entorno en el que los encuestados encontraron por primera vez el uso de drogas fue dentro de su contexto y su propia familia. Los jóvenes crecieron con el consumo de drogas y ocurre abiertamente en el seno de sus familias. Para estos jóvenes, las drogas y el alcohol se perciben como parte de un adulto normal en la familia. De esta manera existen actividades en los integrantes de la familia que intervienen como factores positivos para la formación del significado en los jóvenes. Es así, que ante los actos ajenos, los jóvenes de pandillas puede reforzar su comportamiento. En las narraciones se muestra consumo de drogas en integrantes de la familia y se coloca como un justificación para iniciar el consumo de drogas. ...mi jefe me regaña, cuando ando loco, me golpea, pero le digo ¿que porqué? ¿verdad?, si él también era así.(P5), ...me dice mi hermano, el mayor me dice hazle a la marihuana pero a los químicos no, ya le hago ya, es que la mayoría de mis amigos me dice sobres y por eso le hago (P6).

En algunos casos, el uso normalizado de las drogas dentro del hogar familiar, incluye las experiencias de iniciación con miembros de la familia, con una visión del consumo de drogas como algo común. Sin embargo por otra parte existe una vía más frecuente de inicio en la drogas en estos jóvenes, los amigos de la pandilla. A continuación se describe la forma en que los integrantes de las pandillas influyen en el consumo de drogas.

\section{La influencia de integrantes de la pandilla}

La influencia de los integrantes de la pandilla para el consumo de drogas es descrita por dos tipos de presiones; presión directa y presión indirecta al consumo de drogas.

La presión directa al consumo de drogas se muestra cuando uno de la integrante o el amigo le hace un ofrecimiento real para consumir drogas, se destaca que la mayoría se resiste al ofrecimiento, sin embargo terminan aceptando el consumo, y así comienzan a experimentar los efectos al consumo.... mi amigo dijo que si quería más y dije no mejor no, y luego ya me dio y luego ya me gustó, y esa vez ya me gustó la marihuana y horita ya la pruebo y no me siento nada... es que la mayoría de mis amigos me dice sobres (ofrecen), y por eso le hago y a la vez, me arrepiento (P6).

Es que cuando veo a los demás y así te ofrecen, ni modo de decir que no, después te empiezan a decir cosas (P13).

Aunque el uso de drogas no es una condición de pertenencia, algunos miembros de las pandillas admitieron que probaron drogas por primera vez con el fin de ser aceptados en el grupo. La presión indirecta se muestra en las narraciones de los jóvenes, donde revelan que no existe un ofrecimiento o presión directa entre integrantes de la pandilla, sin embargo, perciben 
que deben de consumir para "sentirse igual que ellos", para "no dejarlos morir", estas declaraciones reflejan de alguna manera los vínculos de amistad y hermandad, así como el valor de solidaridad que existe entre ellos.... pero así, nada más, para no quedarme atrás, de que no, ósea así, para andar igual que ellos, así, dice y riéndose así no se, riéndose, platicando más así por eso, porque fuman todos y yo también quiero fumar para convivir con tus amigos para sentirte igual que ellos, si para sentirme ahí con, ahí con ellos, no se (P4).

Las narraciones anteriores muestran la influencia de los integrantes de la pandilla, las que se pueden explicar por el concepto de la norma subjetiva(13), que menciona que la percepción de normas son el resultado de la evaluación que la persona hace de sí los otros significativos (amigos, padres, hermanos, etc.) quieren que él realice una conducta y de la motivación para complacerlos. Es decir si el joven de la pandilla percibe que sus amigos esperan que consuma, su decisión tenderá a inclinarse hacia el consumo. En relación a esto, la creencia (acertada o equivocada) de que el consumo está ampliamente extendido y aceptado en sus grupos de referencia, ejerce influencia sobre consumir drogas.

\section{Condiciones contextuales del consumo de drogas en pandillas}

En base a sus propias condiciones ambientales, los jóvenes de pandillas buscan los lugares para el consumo y las maneras que facilite el acceso a las drogas. Las condiciones contextuales se describen por el contexto del consumo de drogas y las condiciones desfavorables del barrio.

El contexto del consumo de drogas, se describen por los lugares de consumo y el acceso a la droga. Los lugares comunes de consumo son; "en la plaza", "en la calle", "en la casa de un amigo" y "en las fiestas". Se destaca que de las fiestas, principalmente las quinceañeras son lugares de oportunidad para el consumo de algunas drogas, así como de alcohol....aquí en la plaza, ahí de repente ( $\mathrm{P} 1)$; en la plaza, en la calle. $(\mathrm{P} 12)$, en la casa de uno de los camaradas $(\mathrm{P} 10) ;$...pues a veces los fines de semana, o cuando hay baile aquí con la raza, aquí te invitan y ya por eso le haces a la marihuana y ya así fue en una casa de un camarada (P6); ..llegamos de una quinceañera, estábamos ahí abajo y luego ya eran como las dos de la mañana, sacaron y estaban tomando y sacaron (P13).

Los lugares donde practican el consumo adquieren un sentido trasgresor que va contra la norma $y$ desafía el orden. En este sentido, trae al momento el riesgo al castigo sordeados [escondidos] con la chota [policía] (P16); de tal manera, consumir drogas en los espacios públicos como en las plazas, la calle, en los rincones ocultos de la vía pública, es jugar con el alcance y efectividad de los dispositivos de control. Una investigación ${ }^{(4)}$ explica que en los barrios marginados donde existe un deterioro ambiental y desorganización social, se facilita las actividades de pandillas juveniles incluyendo el consumo de drogas, por la existencia de casas y terrenos abandonados. Así mismo, las fiestas configuran espacios propios de esta juventud que diluyen su alcance y facilitan el despliegue del consumo de drogas.

El acceso al consumo de drogas, se presenta fácil para los jóvenes de pandillas, ya que la oferta de drogas es accesible económicamente y los lugares de acceso forman parte de su contexto, porque existen distribuidores de drogas en el mismo barrio donde viven y los distribuidores las ofrecen en los lugares donde se reúnen los jóvenes. ...no, pues llegan a vendérnosla, viene de repente, y nos venden verdad (se ríe) unos camaradas (P3);...no pues aquí cualquier tipo tiene dinero y donde quiera aquí todos te ofrecen, por que por veinte pesos le dan a uno dos cigarritos o veinticinco, pero ya con eso tienes y ya andas drogado. Bueno aquí nunca ha faltado la marihuana bueno nada más cuando no había, pero siempre, todos los días, todos los días, es más horita ahí tienen, pero sí, siempre, siempre hay (P11).

\section{Significados para el consumo de drogas}

Hasta el momento se ha revisado que el inicio del consumo de drogas en jóvenes de pandillas es por influencia de los integrantes de la pandilla, ya sea por influencia directa y por influencia indirecta. Otra influencia para el consumo de drogas es por la familia, y se explican las condiciones del contexto que facilitan su consumo A través de estas influencias ocurre el inicio de la experimentación del consumo de drogas y al significado del consumo de drogas donde sobresalen actitudes positivas más que actitudes negativas.

\section{Actitudes para el consumo de drogas}

La actitud hacia una conducta, está determinada por las creencias que unen la conducta con los resultados y la evaluación de dichos resultados. Es decir la actitud para el consumo de drogas en los integrantes de pandillas está determinada por el conjunto de creencias sobre las consecuencias del consumo y la evaluación que le atribuyen a tales consecuencias. Sin embargo, estas creencias pueden reforzarse en los jóvenes que ya tienen una experimentación previa de la conducta, lo que los llevará a tener una actitud más favorable o positiva basada no sólo en las creencias individuales sino 
también en la experimentación del consumo de la droga, lo que potencializa la actitud hacia el consumo(14).

Existe una concordancia general que indica que la actitud representa una evaluación acumulable de creencias del sujeto sobre dimensiones atributivas tales como bueno-malo, perjudicial-beneficioso, agradabledesagradable y simpático-antipático. En las narraciones de los sujetos se muestran como sobresalen las actitudes positivas para el consumo de drogas representadas por las ventajas de los efectos placenteros sobre las posibles consecuencias del consumo de drogas (actitud negativa) como símbolo del consumo de drogas.

Sobre la creencia y evaluación del efecto de las drogas en jóvenes de pandillas aparecen las actitudes positivas resaltando imágenes placenteras a través de los diálogos. Por un lado el efecto significa una fuga, una anulación del sentir y el pensar que permite evadir una realidad que angustia: se te olvidan los problemas, se siente más relajado (P8). Por otro lado, el uso de drogas tiene un sentido de estar en el limbo, que permite estar y al mismo tiempo no estar en el mundo, o estar de otra forma: se siente todo más calmadito y como que de repente si se te va la onda (P11). En este registro, el efecto se traduce en una experiencia que toca lo trascendente, significa un pasaje por los rincones de la mente y despierta capacidades ocultas del pensamiento inexplicables: te sientes más acá, y no, es inexplicable la marihuana (P11). El efecto también significa una desinhibición, una liberación del yo que crea estados de excitación y regocijo que le dan al consumo de drogas un sentido festivo y de felicidad: porque ando así muy alegre (P6); te pone la risueña (risas), te da la risueña (P7), en este significado de desinhibición también se muestran efectos mágicos de poder realizar actividades que no se pueden hacer sin el uso de drogas: como que sientes que puedes con todo, te sientes fuerte, te sientes que si te vas a pelear que le vas a ganar (P13).

Por otra parte, los jóvenes también mostraron en las narraciones actitudes con connotaciones negativas de las drogas. ...pues si que está mal lo que hago, pero aparte también porque, pues está mal porque le hace daño a uno, en los pulmones $y$ eso (P4); .. te daña el cerebro, $y$ eso es el problema te hace mal en la cabeza (P7).

Lo anterior refleja que para los jóvenes de pandillas, vivir la sensación es bueno y es malo: lo bueno es la sensación misma del efecto placentero; lo malo, el daño que encierra. La referencia al daño como componente de esta relación demuestra un saber sobre estos riesgos, que a su vez habla del consumo de drogas con las imágenes del deterioro físico y mental condensadas en las del cáncer y el daño neuronal. Pese a lo anterior, no alcanza a opacar los otros discursos que subliman las sensaciones placenteras físicas y mentales del efecto (actitudes positivas). El placer de relajamiento cuando se fuma un cigarrillo, la euforia o la inhibición de los inhalables, el relajamiento y risas que acompaña la vivencia de la marihuana, o lo estimulado que hace sentir en la boca la cocaína, son discursos que los mensajes de las campañas no logran opacar porque circulan por otros canales, unos más cercanos y cotidianos que pasan por el interaccionismo directo de experiencias entre estos jóvenes.

\section{Definiendo las drogas}

Para estos jóvenes, la droga donde se resalta la marihuana se percibe como parte de un adulto normal, se muestra que el punto de comparación para describir la cocaína y los inhalables (solventes: resistol y tolueno) es la marihuana, destacando que son parecidas con los términos "pues es igual" o "empata" en relación a los efectos que estas producen. ...la coca (cocaína), pues es igual como con la marihuana, pero no mas que, pero esta está más fuerte (P10); ...la mota (marihuana) es la vida., nombre es la pura naturaleza, es una planta excelente, es una planta y no es venenosa (se ríe) (P16)....El tolueno (inhalable) no, es pura química, pero empata, pero de plano no le hacemos todos los días, no el tolueno casi no, de vez en cuando, no más cuando no hay mota, es pura química, pero empata (P16).

La definición de cada droga dada por los jóvenes es distinta: unas traen el placer del relajamiento físico (marihuana [mota]), otras la estimulación de los sentidos (tolueno, cocaína [la coca]); pero todas son sensaciones físicas y mentales que se traducen en estar en otro estado o percibir de otra forma y que se destacan por llevar al mismo estado pero empata (P16). La naturaleza de los objetos en este caso las drogas, consiste en el significado que esta encierra para la persona que como tal lo considera(15). El significado que los jóvenes de pandilla le atribuyen a las drogas determina la manera en que ellos están dispuestos a actuar con respecto al mismo y a la forma en la cual se disponen a hablar de ellas.

Otras narraciones muestran que la marihuana para estos jóvenes tiene menos efectos dañinos que el resto de las drogas, por lo que justifican su uso volviéndose una práctica endémica en la pandilla, así mismo esto concuerda con algunos autores, donde muestran que el consumo de marihuana en las pandillas es la de mayor preferencia(10,16). La marihuana en comparación con otras drogas como la cocaína crack o solventes, representa para estos jóvenes una droga con pocos daños a la salud 
y riesgos sociales. ...es que muchos dicen que no es mala como otras drogas, como el resistol que te seca el cerebro y acá y esa cosa no verdad, pero quien sabe como quiera es droga ¿no?, pues como quiera te afecta, pues no sabría explicar en realidad eso. (P9); ...no es que dos o tres acá, dos o tres drogas si te mandan al chorizo verdad pero, pero la mota no, la mota es la vida (P16).

\section{Saber consumir drogas}

Los jóvenes que pertenecen a pandillas describen en sus narraciones el saber consumir drogas en términos de ir adaptándose, controlarla y evitando el bajón. En relación a ir adaptándose al consumo de drogas se refieren en términos de agarrándole el modo y agarre el gusto, así mismo es claro que se van volviendo tolerantes a las sustancias consumidas, dándole un significado de saber consumirlas.

Probar una droga permite saber de ella, pero como todo aprendizaje, no es inmediato: llega con la práctica y pasa por la repetición ....ya después fui agarrándole el modo. Aprender a fumar, aprender a inhalar, a controlar la expulsión del humo o a aguantar en los pulmones el humo de la marihuana, a tomar en las manos el cigarrillo o a dar una baiza es un saber que habla de lo que tiene práctica, de lo que lleva tiempo haciendo y que ha probado de todo, sabe de calidades y tiene experiencia. Es por eso que la edad tiene una relación positiva con el consumo de drogas donde se muestra que los jóvenes de mayor edad tienen mayor consumo (frecuencia y cantidad). Todo este saber sobre las drogas se acumula con el tiempo y viene con la edad. La relación con una droga no es estática: cambia con el tiempo. Ir creciendo significa pasar por etapas en que va variando la cantidad de droga usada o incluso el sentido que se le da a su uso. Con la edad se aprende a controlar la relación con las drogas, a manejar las cantidades adecuadas o la droga propicia para los distintos momentos, en las narraciones sobresale el saber controlarla.

Otro término que aparece como parte del saber consumir drogas es evitar el bajón, las narraciones muestran experiencias de bajón y como los demás integrantes de la padilla interpretan el bajón. ...porque me dio el bajón, porque no le digo que le puse una vez y me sentí bien gacho, se me nublo todo y como que me quiso dar calentura, pero no, está gacho porque pensaba que me iba ir para atrás y ya dije no aquí me voy a morir, no pude controlarlo, me fume como cuatro cigarros (P7).

El grupo banda interpreta el bajón como el límite en la relación con una droga, el exceso también figura una subjetividad: soportar el exceso habla del que tiene experiencia con la droga unos aguantan más. Llegar al extremo del exceso y no resistir sus efectos en sentido negativo los jóvenes lo interpretan como débil y aún le falta se están riendo de ti, de cómo estas, porque saben que acá te dio para bajo la marihuana (P14). En su extremo, el exceso permanente, el constante estado alterado de abuso de una o varias drogas, representa la personalidad del que no sabe manejar su relación con las drogas.

\section{Consideraciones finales}

En el contexto de la vida pandillera de los jóvenes estudiados, el consumo de drogas es una actividad colectiva, y es para muchos, una actividad común. El consumo de drogas se inicia con la influencia de los miembros de la pandilla ya sea por influencia directa o por influencia indirecta, donde se destacan los vínculos de amistad y solidaridad. Así mismo se muestra vinculado el concepto de normas subjetivas, donde el consumo de drogas es percibido como una conducta normativa.

Otra influencia para el consumo de drogas proviene de la familia, donde se observa un consumo de drogas y alcohol por parte de algún integrante de la familia, y existen familiares que apoyan el consumo de drogas. El contexto que facilita la práctica del consumo de drogas en las pandillas es en lugares públicos y fiestas, en particular estos barrios se caracterizan por la existencia de casas y terrenos abandonados lo que facilita la distribución y el consumo de drogas entre los miembros de pandillas. En el inicio del consumo de drogas, en los jóvenes de pandillas se crea el significado del consumo de drogas, donde muestran efectos emocionales y de bienestar, relajamiento, desinhibición y sentimientos de felicidad, donde sobresalen las actitudes positivas más que las actitudes negativas. Las actitudes negativas se refieren al daño donde se demuestra un saber sobre los riesgos, con deterioro físico y mental enfocadas en las del cáncer y el daño neuronal.

En la definición de las drogas, la marihuana en comparación con otras drogas representa para estos jóvenes una droga con pocos daños a la salud y riesgos sociales. La tolerancia física de las drogas es vista como saber consumirlas, ya que describen el saber consumir drogas en términos de ir adaptándose, controlarla y evitando el bajón. El bajón es interpretado como el límite de la relación con una droga.

Para los profesionales del área de la salud, la sugerencia de que las pandillas juveniles son una población vulnerable es una indicación de que estos jóvenes pueden necesitar un acceso especial a los servicios de salud en general y que tienen necesidades de 
atención médica particular como el abuso de sustancias y violencia. Los profesionales de la salud como enfermeros están en la posición de liderar el esfuerzo importante en materia de prevención en jóvenes de pandillas o jóvenes que vivan en zonas marginadas e incentivar el desarrollo de alianzas creativas para tener acceso a estas poblaciones.

\section{Agradecimientos}

Agradecemos a la Comisión Interamericana para el Control del Abuso de Drogas/CICAD de la Secretaria de Seguridad Multidimensional/SSM de la Organización de los Estados Americanos/OEA, la Secretaria Nacional de Políticas sobre Drogas/SENAD do Gabinete de Seguridad Institucional/Brasil, la Escuela de Enfermería de Ribeirao Preto de la Universidad de Sao Paulo y Centro Colaborador de la Organización Mundial de la Salud para el Desarrollo de la Investigación en Enfermería, la población representada en los estudios de investigación, bien como a las autoridades de las universidades representadas por los participantes del Programa En-Line de Especialización en Investigación sobre el Fenómeno de las Drogas, periodos 2006, 2007, 2008 y 2009.

\section{Referencias}

1. Walker-Barnes CJ, Mason CA. delinquency and substance use among gang-involved youth: The moderating role of parenting practices. Am J Comm Psychol. 2004;34(4):235-50.

2. Sanders B, Schneiderman JU, Loken A, Lankenau $\mathrm{SE}$, Bloom JJ. Gang youth as a vulnerable population for nursing intervention. Public Health Nurs. 2009;26(4):346-52.

3. Cerda $P L$, Cerda E, Story L. Violencia y familia en nuevo león. Cienc UANL. 2008;9(1):87-92.

4. Dupéré $V$, Lacourse $E$, Willms $D$, Vitaro F, Tremblay R. Affiliation To youth gangs during adolescence: The interaction between childhood psychopathic tendencies and neighborhood disadvantage. J Abnorm Child Psychol. 2007; 35:1035-45.

5. Howell JC, Decker SH. The Youth gangs, drugs, and violence connection. Washington: US Department of Justice. Office Juvenile Justice Delinquency Prevention Bull 1999;OJJDP Juvenile Justice Bull. [journal on the ibnternet] [acesso 12 jun 2010]. 1999 Jan;NC]171152:1-13. Disponível em: http://www.eric.ed.gov/ PDFS/ED438354.pdf
6. Bennett T, Holloway K. Gangs membership. Drugs and crime in the UK. Br J Criminol. 2004;44(3):305-23.

7. Valdez A, Kaplan C, Cepeda A. The Drugs-violence nexus among mexican-american gang members. J Psychoactive Drugs. 2006;38(2):109-21.

8. Guzmán-Facundo FR, Alonso-Castillo MM. Adquisición do uso de alcohol en un grupo de adolescentes mexicanos: El efecto de la relación con amigos. SMAD, Rev. Eletr Saúde Mental Álcool Drog. (Ed. port.) 2005;1(2). [13 telas]. Disponível em: http://www2.eerp. usp.br/ resmad/artigos.asp

9. Guzmán-Facundo FR, Alonso-Castillo MM, López-García KS. Factores de riesgo y consumo de drogas en bandas juveniles. Desarrollo Cient Enferm. 2006;14(1):409-14. 10. Guzmán-Facundo FR, Pedrão LJ. Personal and interpersonal risk factors in the consumption of ilicit by marginal adolescents and young people from juvenile gangs. Rev. Latino-Am. Enfermagem. 2008;16(3):36874.

11. Strauss A, Corbin J. Basics of Qualitative Research. Techniques and procedures for developing groubded theory. 2.ed. London: Sage Publications; 1998.

12. Secretaria de Salubridad y Asistencia (MX). Reglamento de la ley general de salud en materia de investigación para la salud. México (DF): Porrua; 1987. 13. Ajzen I. The Theory of Planned Behavior. Org Behav Human Decision Process 1991;50: 179-211.

14. Fishbein M, Azjen I. Belief, Attitude and behaviour: an introduction to theory and research. Reading. Massachussets: Addison-Wesley; 1975.

15. Blumer H. Simbolic Interactionism. Perspective and method. Berkeley: University of California; 1969.

16. MacKenzie K, Hunt G, Joe-Laidler K. Youth gangs and drugs: The case of marijuana. J Ethn Subst Abuse. 2005;4(3-4):99-134. 